\title{
Turbulent pair dispersion as a continuous-time random walk
}

\author{
Simon Thalabard, Giorgio Krstulovic \\ and Jérémie Bec $\dagger$ \\ Laboratoire Lagrange UMR 7293, Université de Nice-Sophia Antipolis, \\ CNRS, Observatoire de la Côte d'Azur, Bd. de l'Observatoire, 06300 Nice, France.
}

(Received 24 August 2021)

The phenomenology of turbulent relative dispersion is revisited. A heuristic scenario is proposed, in which pairs of tracers undergo a succession of independent ballistic separations during time intervals whose lengths fluctuate. This approach suggests that the logarithm of the distance between tracers self-averages and performs a continuous-time random walk. This leads to specific predictions for the probability distribution of separations, that differ from those obtained using scale-dependent eddy-diffusivity models (e.g. in the framework of Richardson's approach). Such predictions are tested against highresolution simulations and shed new lights on the explosive separation between tracers.

\section{Introduction}

Tracers in a turbulent flow separate in an explosive manner. Their averaged square distance becomes independent of their initial separation and grows as $t^{3}$ at large times. This explains the ability of turbulence to considerably enhance mixing (Dimotakis 2005), but also links to fundamental issues in turbulence, where a key question is to relate the irregularity of the Lagrangian flow with the persistence of a finite dissipation at infinite Reynolds numbers (Cardy et al. |2008, Eyink \& Drivas 2014). Since the first observation of the $t^{3}$ law by Richardson (1926) and its interpretation in terms of Kolmogorov's similarity hypothesis by Obukhov (1941), precise experimental and numerical measurements and acute modelling of pair separation have proven to be a particularly laborious exercise, as stressed for instance in the reviews by Sawford (2001) and Salazar \& Collins (2009).

A difficulty in observing the explosive law stems from the huge separation of timescales that it requires. Batchelor (1950) indeed showed that the $t^{3}$ law is preceded by a ballistic regime during which the mean-square separation is $\propto t^{2}$. This dominates relative dispersion as long as the initial velocity difference between the tracers has not changed much, that is up to times of the order of the eddy turnover time $\tau_{r_{0}} \propto r_{0}^{2 / 3}$ associated to the inertial-range initial separation $r_{0}$ - hereafter referred to as Batchelor's timescale. The explosive $t^{3}$ law takes over at times $t \gg \tau_{r_{0}}$. While Batchelor's predictions are quantitatively confirmed in particle-tracking experiments (Berg et al. 2006 : Ouellette et al. 2006) and in direct numerical simulations (Yeung 1994: Sawford et al. 2008: Bitane et al.|2012), the most manifest observations of the $t^{3}$ law are limited to initial separations $r_{0}$ close to the Kolmogorov dissipative scale $\eta$ (Ott \& Mann 2000, Boffetta \& Sokolov 2002, Biferale et al. 2005; Eyink 2011). For particles whose initial separation lies in the inertial range, the $t^{3}$ growth is more elusive and emerges at best as a short transient on times both much larger than $\tau_{r_{0}}$ and smaller than the integral timescale, from which the separation between tracer particles becomes purely diffusive. Even though the $t^{3}$ explosive law can be

$\uparrow$ Email address for correspondence: jeremie.bec@oca.eu 
understood on purely dimensional grounds, there is today a lack of sufficiently accurate data that would substantiate the $t^{3}$ law and constrain the underlying mechanisms.

Long-established modelling of relative dispersion is based on the assumption that distances between tracers undergo a scale-dependent diffusion (Richardson 1926). As stressed by Falkovich et al. (2001), this presupposes to observe the system on a timescale $t$ much longer than the Lagrangian correlation time $\tau_{r}^{\mathcal{L}}$ of the velocity difference between tracers at a distance $r$. For separations of the order of the typical separation $r \sim \varepsilon^{1 / 2} t^{3 / 2}$ (with $\varepsilon$ denoting the mean kinetic energy dissipation rate of the turbulent flow), this presumes that $\tau_{r}^{\mathcal{L}} \ll \varepsilon^{-1 / 3} r^{2 / 3} \sim \tau_{r}$ and thus leads to the unrealistic assumption that the Lagrangian correlation time is much shorter than the Eulerian turnover time. Markovian models involving the joint evolution of separations and velocity differences have been introduced to circumvent such drawbacks (Kurbanmuradov \& Sabelfeld 1995, Thomson \& Wilson 2013). They are mainly based on the shortness of acceleration correlation times in turbulence and usually rely on the input of Eulerian single-time statistics. Possible pitfalls in their justification relate to neglecting long-term memory effects due to the persistence of turbulent flows. In an ideal infinite-Reynolds number turbulent flow, most Markovian models admit scaling solutions of the form $r \propto t^{3 / 2}$ and $v=(\mathrm{d} r / \mathrm{d} t) \propto t^{1 / 2}$. However, at the same time, a small-scale regularization is usually required in order to prevent particle pairs from collapsing together at a finite time with a vanishing velocity difference. The scaling solutions depicted above are usually coexisting with their "dual" $r \propto\left(t_{\star}-t\right)^{3 / 2}$ with $v \propto-\left(t_{\star}-t\right)^{1 / 2}$. On the one hand, preventing possible finite-time singularities requires modelling the dissipative-range physics of turbulence. On the other hand, such events might correspond to the loss of memory that could justify the applicability of diffusive approaches at sufficiently large times. The recent numerical studies of relative dispersion by Scatamacchia et al. (2012) and Bitane et al. (2013) have clearly stressed the importance of small scales on the overall evolution of distances: Some pairs remain trapped at scales $r \ll r_{0}$ for very long times and give an important contribution to the average separation. Accounting for such trapping events has motivated the introduction of non-Markovian models (Shlesinger et al. 1987, Faller 1996, Rast \& Pinton 2011). Most of them rely on prescribing a distribution of waiting times.

We follow here a slightly different route to account for non-Markovianity. Numerical data from a large-scale simulation of 3D homogenous turbulence are used, to question the possibility that systematic deviations for the distribution of the inter-particle separations from Richardson's self-similar solution could stem from a multiplicative process. We motivate this possibility using a handwaving description of a ballistic phenomenology, observed to be compatible with the bulk of the distribution of distances. We then flesh this observation and describe a piecewise-ballistic phenomenological toy model, which yields a whole family of self-similar distributions for the inter-particle distances, and predict that the distributions of their logarithms should collapse towards a well-defined distribution. This prediction is then tested against numerical data.

\section{The inter-particle distance as a multiplicative process?}

Most models for relative dispersion reproduce the $t^{3}$ explosive law fo the long-time behaviour of the mean-square separation. However, they usually yield different probability distributions of the distance $r$ between tracers at time $t$. Richardson's eddy-diffusivity approach leads to the self-similar distribution (see Salazar \& Collins 2009)

$$
p(r, t)=\frac{426}{35} \sqrt{\frac{286}{\pi}} \frac{r^{2}}{(g \varepsilon)^{3 / 2} t^{9 / 2}} \exp \left[-\frac{1}{2} \sqrt[3]{1287} \frac{r^{2 / 3}}{(g \varepsilon)^{1 / 3} t}\right],
$$


which is uniquely determined by Richardson's constant $g=\left\langle r^{2}\right\rangle /\left(\varepsilon t^{3}\right)$. The only settings where this distribution is exact is for delta-correlated-in-time flows (Falkovich et al. 2001). Be it using massive numerical simulations or sophisticated particle-tracking experiments, one expects to measure some systematic deviations from this distribution. Those deviations exist and have been reported but, quite remarkably, they are rather mild: Richardson's diffusive mechanism appears to predict correctly the bulk of the distribution and only fails to accurately describe rare events. More precisely, for tracers with an initial distance $r_{0}$ of the order of the Kolmogorov scale, Richardson's distribution seems to overestimate the fraction of particles separating faster than $t^{3}$ (Scatamacchia et al. 2012). When $r_{0}$ lies within the inertial range, it underestimates the fraction of pairs that separate faster than the average (Bitane et al.|2013). As those deviations are very fine, one cannot preclude that they are either a consequence of intermittency or of finite-Reynolds-number effects that would contaminate the statistics at both very large and very small scales. A more serious concern exists, though: As stated earlier, the eddydiffusivity framework entails short correlation times for the velocity differences between tracers and has thus debatable physical origins.

In order to remain closer to phenomenological considerations, we propose a completely different mechanism that relies on Batchelor's ballistic separation of pairs. Let us imagine the following simplified scenario. Two tracers initially separated by a distance $r_{0}$ inside the inertial range will follow a ballistic motion with a velocity difference $\delta u_{0}$ for a short period of time $\tau_{0}$. By Kolmogorov 1941 phenomenology the time and velocity should scale as $\tau_{0} \sim r_{0}^{2 / 3}$ and $\delta u_{0} \sim r_{0}^{1 / 3}$. Therefore, after the time $\tau_{0}$ the pair will be separated by a distance $r_{\tau_{0}}=r_{0}+\tau_{0} \delta u_{0}=r_{0}\left(1+a_{0}\right)$, where $a_{0}$ is in principle a scale-independent random variable that accounts for the fluctuations of the flow. Applying the same argument to $r_{\tau_{0}}$ we find $r_{\tau_{0}+\tau_{1}}=r_{0}\left(1+a_{0}\right)\left(1+a_{1}\right)$ where $a_{1}$ is independent of $a_{0}$, and so on. Finally, the relative distance between the two tracers at time $t$ is given by $r_{t}=r_{0}\left(1+a_{0}\right) \cdots(1+$ $a_{n}$ ) with $t=\tau_{0}+\cdots+\tau_{n}$, where by construction the number of terms is $n \sim \ln t$. Although naive, such a piecewise-ballistic phenomenology relies on the physical ideas of Batchelor and makes use of the ballistic separation between tracers whose validity is strongly assessed by numerics and experiments. This scenario suggests that the $t^{3}$ law may appear as the consequence of the separations increasing multiplicatively rather than additively. If such was indeed the case, the natural observable is not the distance but rather its logarithm, which is then expected to self-average. Indeed all the $n$ random variables $a_{i}$ are independent and identically distributed. By the law of large numbers, we thus expect at large times $\ln r_{t} \propto n \sim \ln t$.

To test those ideas we analyse data from a large direct numerical simulation of 3D homogeneous turbulence inside a triply-periodic box, which uses $4096^{3}$ grid points to achieve a Taylor-based Reynolds number $R_{\lambda} \simeq 730$ and is seeded with $10^{7}$ tracers (see Bitane et al. 2013, for more details). Figure 1(a) shows the evolution of the average of the logarithm $\rho=\ln \left(r_{t} / r_{0}\right)$ of the inter-particle separations, together with its standard deviation. The statistics are conditioned on the initial distances $r_{0}$ between the particles,

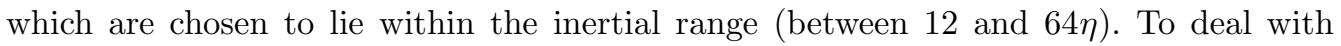
dimensionless quantities, distances between pairs of tracers are divided by their initial separations, while timescales are divided by the associated Batchelor time scale. At large times the averaged logarithm of the separation exhibits a slant asymptote, whose linear slope cannot be quantitatively distinguished from $3 / 2$ : The dashed line shows a behaviour $\langle\rho\rangle \approx(3 / 2) \ln t+$ const. From the law of large numbers, one also expect that the variance $\left\langle\delta \rho^{2}\right\rangle=\left\langle\rho^{2}\right\rangle-\langle\rho\rangle^{2}$ increases as $\ln t$. This feature is clearly not observed: The standard deviation of the logarithm rather reaches a plateau for times much longer than the 

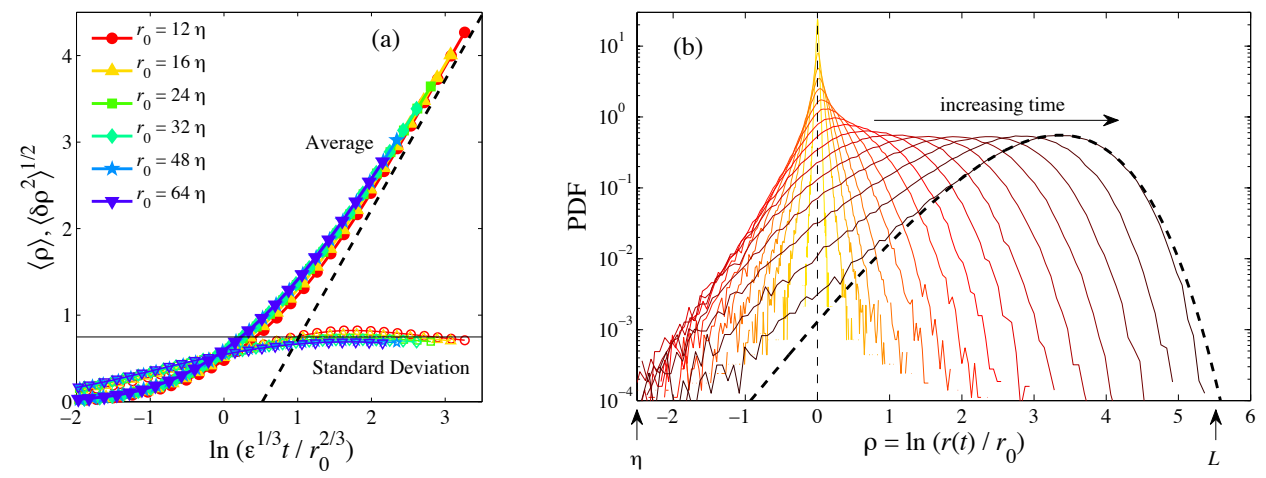

Figure 1. (Colour online) (a) Average value and standard deviation of $\rho=\ln \left(r(t) / r_{0}\right)$ for various initial separations $r_{0}$ and as a function of $\ln \left(t / \tau_{r_{0}}\right)$ with $\tau_{r_{0}}=\varepsilon^{-1 / 3} r_{0}^{2 / 3}$. The dashed line is $\langle\rho\rangle=(3 / 2) \ln \left(t / \tau_{r_{0}}\right)+(1 / 2) \log g-0.46$ with $g=0.52$ and the solid line $\left\langle\delta \rho^{2}\right\rangle^{1 / 2}=0.748$; both correspond to Richardson's self-similar predictions. (b) Distributions of $\rho$ for $r_{0}=12 \eta$ and various times; the two vertical arrows mark the dissipative scale $\eta$ and the integral scale $L$. The dashed line is Richardson's distribution.

Batchelor time $\tau_{r_{0}}$. From the latter observation, we cannot exclude that the evolution of the inter-particle distances be multiplicative. Yet, such a multiplicative evolution would need to be non-Markovian. In other words a non constant time stepping may be involved in the above phenomenology. This would break the assumptions of the law of large numbers.

Figure 1 (b) shows the time evolution of the probability distribution of $\rho$. After a short transient, one observes a self-similar regime, which might explain the behaviours of both the average and the standard deviation. Indeed, if the distribution of distances takes the scale-invariant form $p(r, t)=r^{-1} \Phi[r / \ell(t)]$, with $\ell(t)$ an arbitrary function of time, then the distribution of $\rho=\ln \left(r / r_{0}\right)$ can be written $p(\rho, t)=\Psi\left[\rho-\log \left(\ell(t) / r_{0}\right)\right]$, with $\Psi[z]=\Phi\left[\mathrm{e}^{z}\right]$. This leads to

$$
\langle\rho\rangle=\ln \left(\ell(t) / r_{0}\right)+\langle z\rangle, \quad\left\langle\delta \rho^{2}\right\rangle=\left\langle\delta z^{2}\right\rangle
$$

where the (time-independent) moments of $z$ are obtained by using the distribution $\Psi[z]$. Note that by construction $\langle\log r\rangle$ does not depend on the initial conditioning $r_{0}$. If in addition $\ell(t)$ is a power-law of $t$, then $\langle\rho\rangle \propto \ln t$ and $\left\langle\delta \rho^{2}\right\rangle \simeq$ const, as observed in the data. For Richardson distribution (2.1), $\ell(t)=(g \varepsilon)^{1 / 2} t^{3 / 2}$ and, remarkably, the full distribution of $z$ depends neither on the constant $g$, nor on any other physical quantities. Hence, once $\langle\rho\rangle$ is known, the distribution of $\rho$ induced by 2.1 does not require any fitting. The behaviours associated to Richardson's distribution are displayed in Figure 1(a) - dashed and solid lines. They give a good approximation for both the mean and the standard deviation of $\rho$. As seen in Figure 1(b), after the transient given by the Batchelor timescale, Richardson's distribution reproduces well the bulk but fails to accurately predict the extreme fluctuations of $\rho$. This can hardly be blamed on finite-range effects as almost all separations are well inside the inertial range - as indicated by the two vertical arrows.

The most noticeable departure from Richardson's distribution occurs at separations much smaller than their average. We indeed clearly observe $p(\rho) \propto \mathrm{e}^{2 \rho}$, hereby evidencing a power-law behaviour $p(r) \propto r$ for distances (as already observed by Bitane et al. 2013). This contrasts to the scaling $p(r) \propto r^{2}$ obtained from eddy-diffusivity models in the K41 framework, including in addition to Richardson's model those where the diffusion coefficient depends also on time (i.e. of the form $D \sim \varepsilon^{\alpha} r^{\beta} t^{\gamma}$, see Hentschel \& 
Procaccia 1984). This suggests that the statistics of extreme events cannot be captured by simple diffusive models. However, as we will now see, the heuristic multiplicative approach described earlier can be refined. It leads to a qualitative understanding of the self-similarity and the tails of the distributions observed in Figure 1(b).

\section{A piecewise-ballistic heuristic scenario}

A model that predicts the long-time $t^{3}$ explosive law but disregards the short-time ballistic behaviour may yield some biased insights, as it will probably fail to take into account the intrinsic non-Markovian nature of pair separation. Ilyin et al. (2010) and Eyink \& Benveniste (2013) therefore choose to study pair dispersion in terms of a diffusion equation with a memory kernel. We explore here an alternative scenario where we literally implement the ballistic ideas. We argue that this short-time behaviour can be thought of not only as a transient feature but as the central ingredient, that yields the explosive regime. To this end, we propose a piecewise-ballistic scenario of tracers separation, described in terms of a toy stochastic model which fits into the general class of so-called Continuous-Time Random Walks (CTRW, see Hughes 1995). The model is rooted on a very intuitive phenomenology, which is in a sense built in to yield the $t^{3}$ law. Yet, it also captures non-trivial features of the large-time statistics of the separations between tracers, among which their self-similarity, their explosive nature, and a qualitative description of the distributions of extreme events.

\subsection{Intuitive description of a stochastic piecewise-ballistic model}

Given a pair of tracers, we denote $\mathbf{r}(t)$ and $\delta \mathbf{u}(t)$ their separation and relative velocity, respectively. A "ballistic modelling" consists in assuming that during a time-lapse $\tau$, the velocity $\delta \mathbf{u}(t)$ remains constant, so that $\mathbf{r}(t+\tau) \simeq \mathbf{r}(t)+\tau \delta \mathbf{u}(t)$. As demonstrated by Bitane et al. (2012) for separations inside the inertial range, the ballistic motion holds typically for a time $\tau$ of the order of the time needed to damp out $|\delta \mathbf{u}(t)|^{2}$ with the average turbulent dissipation rate $\varepsilon$, namely $\tau \simeq|\delta \mathbf{u}(t)|^{2} /(2 \varepsilon)$. Applying recursively this heuristic argument suggests that the separation between the two tracers undergoes a sequence of non-correlated ballistic increases or decreases at times $t_{0}=0, t_{1}, t_{2} \ldots t_{k}$ (see Figure 2 Left). Defining the origin of time such that $\mathbf{r}(0)=\mathbf{r}_{0}$, the separation $\mathbf{r}_{k}=\mathbf{r}\left(t_{k}\right)$, and corresponding time $t_{k}$ will then evolve jointly as

$$
\mathbf{r}_{k+1}=\mathbf{r}_{k}+\frac{\left|\delta \mathbf{u}_{k}\right|^{2}}{2 \varepsilon} \delta \mathbf{u}_{k} \quad \text { and } \quad t_{k+1}=t_{k}+\frac{\left|\delta \mathbf{u}_{k}\right|^{2}}{2 \varepsilon} .
$$

In the language of CTRW, the values $\left(\mathbf{r}_{k}, t_{k}\right)$ are called the turning points of the process. Between two successive turning points, the separation is by construction ballistic, so that its value at any time can be obtained by linear interpolation. This choice corresponds to a leaping CTRW, as already used by Shlesinger et al. (1987) in the context of a Levy walk description of turbulent pair dispersion. To entirely describe the statistics of the separations, one now only needs to prescribe statistics $(i)$ for the moduli of the relative velocities $\left|\delta \mathbf{u}_{k}\right|$, and (ii) for the longitudinal components of the velocities $\delta \mathbf{u}_{k}^{\|}=$ $\delta \mathbf{u}_{k} \cdot \mathbf{r}_{k} /\left|\mathbf{r}_{k}\right|$. In principle the distribution of turbulent velocity differences non-trivially depends on the scale $\left|\mathbf{r}_{k}\right|$. This can be due to finite-size effects, so that one has to distinguish between dissipative, inertial, and integral scales. We focus here on separations that always remain within the inertial range and exclude any intermittent corrections to K41 similarity hypothesis. While crude, such an assumption will prove to be sufficient to reproduce the main mechanisms of relative dispersion. In this framework, the relevant 

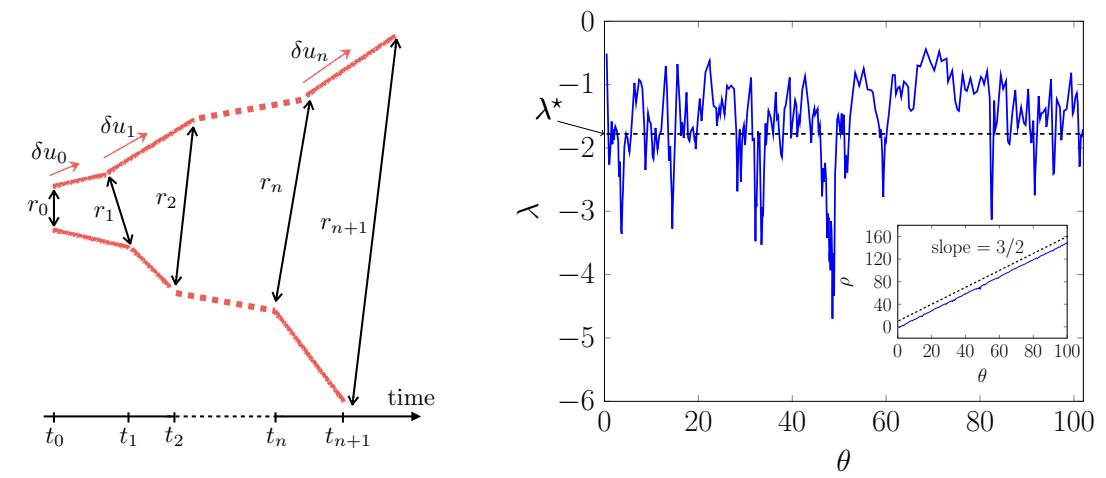

Figure 2. (Color online) Left: Sketch of a piecewise-ballistic scenario. Right: A typical realization of $\lambda$ as a function of the physical logarithmic time $\theta$, and the corresponding evolution of $\rho=\ln \left(r / r_{0}\right)$ (inset). Here, $\alpha$ is uniform between -1 and 1 , and $\log \beta$ is Gaussian with zero mean and unit variance (Please see the text for the definitions).

inputs are the statistics of

$$
\alpha_{k}=\frac{\delta \mathbf{u}_{k}^{\|}}{\left|\delta \mathbf{u}_{k}\right|} \quad \text { and } \quad \beta_{k}=\frac{\left|\delta \mathbf{u}_{k}\right|^{3}}{2 \varepsilon\left|\mathbf{r}_{k}\right|} .
$$

The variables $\alpha_{k} \in[-1,1]$ should have an asymmetric distribution in order to reproduce the skewness of longitudinal velocity differences in turbulence. The variables $\beta_{k}$ account for the fluctuations in the rate of energy transfer and, under K41 assumptions, should be independent of the $\mathbf{r}_{k}$ 's. The time lapses between two consecutive turning points may be thought of as correlation times: It is then natural to prescribe that both $\alpha_{k}$ and $\beta_{k}$ be independently distributed. We later refer to the distributions of the noises $\alpha_{k}$ and $\beta_{k}$ as $\alpha$ and $\beta$ - without a subscript - and denote with $\langle\cdot\rangle$ the average over their realizations.

Under these assumptions the dynamics of the distance $r_{k}=\left|\mathbf{r}_{k}\right|$ reduces to

$$
r_{k+1}=r_{k}\left(1+2 \alpha_{k} \beta_{k}+\beta_{k}^{2}\right)^{1 / 2} \quad \text { and } \quad t_{k+1}=t_{k}+(2 \varepsilon)^{-1 / 3}\left(\beta_{k} r_{k}\right)^{2 / 3} .
$$

Note that this process is not purely multiplicative. As discussed in the previous section, if the time increments $\tau_{k}=t_{k+1}-t_{k}$ were constant, then the distributions of the logarithms of the distance at a given observation time would evolve towards a Gaussian distribution given by the Central Limit Theorem. This is however not the case here, as the time associated to a given pair of tracers is itself a random variable, which is neither additive nor multiplicative. In the present paper, we do not need to prescribe further the distributions of $\alpha$ and $\beta$. The only constraint concerns the quantity $\left\langle\ln \left(1+2 \alpha \beta+\beta^{2}\right)\right\rangle$. It is required to be positive in order to ensure that the times $t_{k}$ go to infinity as the number of turning points diverges. This prevents the sequence $t_{k}$ from converging and the two tracers from touching each other in a finite time.

\subsection{Statistics of the separations from the piecewise ballistic perspective}

We shall not here attempt to work out in full mathematical details the statistics of the separations which the model predicts. Rather, we focus on a general and qualitative description of those, based on simple physical arguments.

\subsubsection{Self-Similarity}

The piecewise-ballistic scenario as modeled by the system 3.3 yields a whole family of self-similar distributions for the separations. To understand the origin of this self- 
similarity, it is convenient to introduce the non-dimensional "logarithmic" variables

$$
\theta_{k}=\ln \left(t_{k} / \tau_{0}\right), \quad \rho_{k}=\ln \left(r_{k} / r_{0}\right), \quad \text { and } \quad \lambda_{k}=\ln \left(r_{k} / r_{0}\right)-(3 / 2) \ln \left(t_{k} / \tau_{0}\right),
$$

where $\tau_{0}=(2 \varepsilon)^{-1 / 3} r_{0}^{2 / 3}$ is a characteristic time lapse associated to the initial separation $r_{0}$. The largest values of $\lambda$ indicate pairs that separate faster than the typical explosive separation, while large negative values are obtained when they get close to each other. We next define $p_{\theta}(\rho)$ the probability density of $\rho$ conditioned on $\theta$, regardless of the number of turning points needed to reach this time, and write $\langle\cdot\rangle_{\theta}$ the corresponding average.

Self-similarity is achieved if the statistics of the variable $\lambda_{k}$ become steady at long times, e.g. when $\theta \gg 1$. A rigorous proof that $\lambda_{k}$ evolves towards a stationary distribution $p_{\infty}$ goes beyond the scope of the present paper. Still, let us give qualitative arguments, starting from the evolution of $\theta_{k}$ and $\lambda_{k}$. The combination of (3.1) and (3.4) yields

$$
\lambda_{k+1}=\lambda_{k}+\frac{3}{2} \ln \frac{\left(1+2 \alpha_{k} \beta_{k}+\beta_{k}^{2}\right)^{\frac{1}{3}}}{1+\beta_{k}^{\frac{2}{3}} \mathrm{e}^{\frac{2}{3} \lambda_{k}}} \quad \text { and } \quad \theta_{k+1}=\theta_{k}+\ln \left(1+\beta_{k}^{\frac{2}{3}} \mathrm{e}^{\frac{2}{3} \lambda_{k}}\right) .
$$

The equation for $\lambda_{k}$ is closed as it does not involve the time variable $\theta_{k}$. A typical realization of the $\lambda_{k}$ 's is shown in Figure 2 (Right). They fluctuate around the specific value $\lambda^{\star}$, univocally defined by

$$
\left\langle\ln \left(1+2 \alpha \beta+\beta^{2}\right)\right\rangle=3\left\langle\ln \left(1+\beta^{2 / 3} e^{2 \lambda^{\star} / 3}\right)\right\rangle .
$$

It is easily shown that $\lambda^{\star}$ is always negative. The evolution of $\lambda_{k}$ can then be decomposed as the sum of a noise $\mathcal{W}$ with zero mean and a restoring stochastic force $\mathcal{F}$, namely

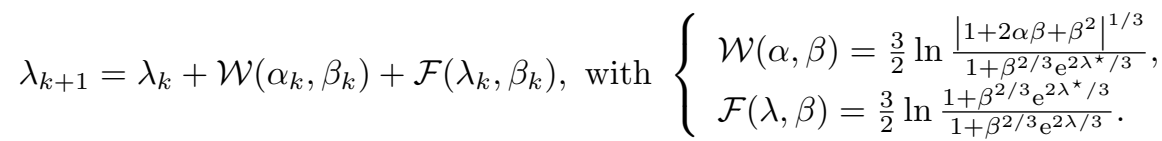

The force $\mathcal{F}(\lambda, \beta)$ is a decreasing function of $\lambda$ and changes sign at $\lambda=\lambda^{\star}$. We therefore expect $\lambda^{\star}$ to be a recurrent point. This is the source of the stationarity of the process $\lambda_{k}$, which we have observed numerically. The stationarity of $\lambda_{k}$ has several consequences. First, the average logarithmic separation grows asymptotically when $\theta \gg 1$ as $\langle\rho\rangle_{\theta}=$ $3 \theta / 2+\langle\eta\rangle_{\infty}+\mathrm{o}(1)$ (see inset of Figure 2 Right), while its variance becomes constant, as observed earlier in our data. Second, the stationarity of $\lambda_{k}$ implies an explosive behaviour of separations. The initial separation $r_{0}$ does not appear in the dynamics (3.5). Also, one can easily see that the specific choice of $\tau_{0}$ ensures that $r_{0}$ can be simplified in the definition of $\lambda_{k}$. The dependence upon the initial separation is thus entailed in the definition of $\theta_{k}$. However, this dependence disappears when $t \rightarrow \infty$, so that the stationary distribution of $\lambda_{k}$ is independent of $r_{0}$. Finally, for large-enough times the probability density of the logarithmic separations is simply translated by the dynamics around its average value: $p_{\theta}(\rho)=p_{\infty}(\rho-3 \theta / 2)$. This implies that, at large times, the statistics of the distance $r$ approach the self-similar form $p(r, t) \simeq r^{-1} p_{\infty}\left[\ln \left(r /\left(\varepsilon^{1 / 2} t^{3 / 2}\right)\right)\right]$, which is independent of $r_{0}$.

\subsubsection{Tails}

The piecewise ballistic mechanism does not yield a single but a whole family of selfsimilar distributions $p_{\infty}$, depending on the choices of $\alpha$ and $\beta$. We can however try to characterize the tails of $p_{\infty}$.

The right-end tail $\left(\lambda>\lambda^{\star}\right)$ corresponds to tracers that separate faster than the average. At large times, the piecewise model (3.5) predicts that $\lambda(\theta)$ is negative, or in other words, 
that the logarithmic distance $\rho$ is strictly smaller than three halves of the logarithmic time. It is indeed easily checked that if $\lambda$ is negative at a given turning point, then it remains negative afterwards. As $\lambda$ winds up fluctuating around $\lambda^{\star}<0$, it is almost surely negative at large times. A noticeable consequence is that the limiting self-similar distributions have a right-end cutoff. Such a behaviour contrasts that obtained from eddy-diffusivity models (including Richardson's), whose tails fall as a double exponential at large values.

The other tail $(\lambda \rightarrow-\infty)$ captures the statistics of pairs that are not separated. Numerical simulations of the model reveal that its form does strongly depend on the distribution of $\alpha$ and $\beta$. To understand further this behaviour, let us define $\Gamma=\langle\mathcal{F}(-\infty, \beta)\rangle$ and $\kappa=\left\langle\mathcal{W}(\alpha, \beta)^{2}\right\rangle^{1 / 2}$, and describe two asymptotic regimes $\kappa \ll \Gamma$ and $\kappa \gg \Gamma$.

(i) $\kappa \ll \Gamma$ describes a situation where tracers almost never come close to each other. When they do so, they are immediately pulled back to their initial separation by the restoring force $\mathcal{F}$. In this case, one-step excursions dominate the left-end tail of the statistics, which is therefore entirely determined by the distribution of $\mathcal{W}$.

(ii) The case $\Gamma \ll \kappa$ is opposite. Here, two particles need to undergo a large number of ballistic steps to be pulled back towards $\lambda^{\star}$. The time of each step is in average smaller than typical correlation time $\Delta \theta^{\star}=\left\langle\log \left(1+\beta^{2 / 3} \mathrm{e}^{2 \lambda^{\star} / 3}\right)\right\rangle$ at $\lambda=\lambda^{\star}$. In this limit, the noise is dominant and the discrete dynamics can be approximated by a Brownian motion with a positive drift. One can check that this yields a stationary distribution whose left-end tail is $\propto \mathrm{e}^{(2 / 3+\gamma) \lambda}$ where $\gamma>0$ depends on the noise kernels. With an accurate choice of the noises, the left exponential slope is therefore likely to be shallower than Richardson's left exponential slope of 3, as suggested by the numerical data.

\section{Scaling of the distribution of distances}

At this point, one may wonder whether the piecewise-ballistic phenomenology achieves a better description of the full statistics than Richardson's distribution. As the precise shape of the statistics obtained from the piecewise-ballistic scenario are noise-dependent, a detailed answer would require to plug into our stochastic model some "realistic noises" for the distributions of $\alpha$ and $\beta$. This goes beyond our intention, since we consider here the set of equations (3.1) as a "scenario" rather than a genuine "modelling" of the separations.

To our taste, the main virtue of the model is that it provides a non-Markovian physical interpretation to the explosive nature of the inter-particle separations, viz., the independence upon the initial separation. We believe that dynamical memory effects are essential for the understanding of extreme events in relative dispersion. One may therefore wonder whether such effects are universal and whether their signature is intrinsically linked to the explosive separation. A related question concerns the origin of the observed deviations to Richardson's self-similar distribution. Are they due to finite-size effects, to the intermittency of velocity statistics, or rather, as we think, to the limits of the eddydiffusivity approach? Our impression is that this question has been somehow overlooked in the previous literature. Experimental and numerical datasets are often confronted to Richardson's distribution using a linlog representation of the distance neighbour function $p(r, t) /\left(4 \pi r^{2}\right)$ in such a way that Richardson's distribution appears as a straight line (see, e.g., Ouellette et al. 2006). Such a representation puts a visual emphasis on the collapse of the bulk of the distribution, but is not optimal for a thorough study of the tails. In Figure 3, we use linlog coordinates to represent the distributions of the rescaled logarithmic distances $\tilde{\rho}=[\rho-\langle\rho\rangle] /\left[\left(\left\langle\rho^{2}\right\rangle-\langle\rho\rangle\right)^{1 / 2}\right]$ observed in the numerical simulation for six different initial separations $r_{0}$ inside the inertial range and for each at 


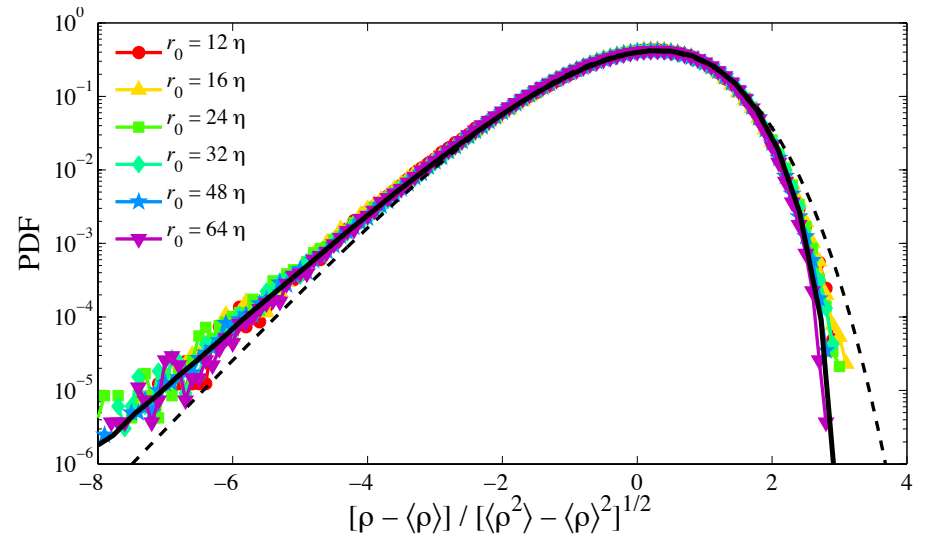

Figure 3. (Color online) Probability density function of the logarithm of the inter-particle distance $\rho=\ln \left(r(t) / r_{0}\right)$ for various $r_{0}$ and, in each case, at time $t \approx 9 \tau_{r_{0}}$ with $\tau_{r_{0}}=\varepsilon^{-1 / 3} r_{0}^{2 / 3}$. The dashed line is Richardson's distribution. The black solid line is the steady distribution obtained numerically from the ballistic model for $\ln \beta \sim \mathcal{N}(0,1)$ and $p(\alpha)=(5 / 6)((\alpha+1) / 2)^{-1 / 3}$.

the fixed time $t \approx 9 \tau_{r_{0}}$. With such a choice, the distribution of the logarithmic distances seems to converge towards a single distribution, regardless of $r_{0}$. The collapse of the full distribution makes the explosive nature of pair separation explicit. It is also once again clear that the limiting distribution is not Richardson's (dashed line). Note that a casual choice for the statistics of $\alpha$ and $\beta$ makes the piecewise ballistic steady distribution (solid line) fit the data better. The model predicts a sharp cutoff at large values. However, the current statistical accuracy does not enable us to discriminate between such a behaviour and the double exponential obtained in the framework of eddy-diffusivity approaches.

To conclude, let us stress again that the piecewise-ballistic phenomenology provides a new and intuitive way of thinking about the problem of pair dispersion and reproduces some salient statistical features of tracer separation. While it might also be used to investigate possible effects of the fluid flow intermittency, we limited here our study to the K41 framework. The proposed toy model displays a number of general trends that include $(i)$ the explosive nature of the statistics, or in other words the property that the steady distributions do not depend on the initial separation; (ii) their selfsimilarity, which makes the statistics of the logarithm of the separation collapse towards a single distribution; (iii) the presence of a right-end cutoff in the associated probability density; $(i v)$ the growth of the average of the logarithmic separation as three halves of the logarithmic time, compatible with the $t^{3}$ law, resulting from the multiplicative nature of the separation process.

This research has received funding from the European Research Council under the European Community's Seventh Framework Program (FP7/2007-2013 Grant Agreement No. 240579) and from the French Agence Nationale de la Recherche (Programme Blanc ANR-12-BS09-011-04).

\section{REFERENCES}

BATChelor, G.K. 1950 The application of the similarity theory of turbulence to atmospheric diffusion. Quart. J. R. Meteorol. Soc. 76, 133146.

Berg, J., Lüthi, B., Mann, J. \& Otт, S. 2006 Backwards and forwards relative dispersion in turbulent flow: An experimental investigation. Phys. Rev. E 74, 016304.

Biferale, L., Boffetta, G., Celani, A., Devenish, B.J., Lanotte, A. \& Toschi, F. 2005 
Lagrangian statistics of particle pairs in homogeneous isotropic turbulence. Phys. Fluids 17, 115101.

Bitane, R., Homann, H. \& Bec, J. 2012 Timescales of turbulent relative dispersion. Phys. Rev. E 86, 045302.

Bitane, R., Homann, H. \& Bec, J. 2013 Geometry and violent events in turbulent pair dispersion. J. Turb. 14 (2), 23-45.

Boffetta, G. \& Sokolov, I.M. 2002 Relative dispersion in fully developed turbulence: The richardson's law and intermittency corrections. Phys. Rev. Lett. 88, 094501.

Cardy, J., Falkovich, G. \& Gawedzki, K. 2008 Non-equilibrium statistical mechanics and turbulence, Lond. Math. Soc. Lect. Note Ser., vol. 355. Cambridge University Press.

Dimotakis, P.E. 2005 Turbulent mixing. Annu. Rev. Fluid Mech. 37, 329-356.

Eyink, G.L. \& Drivas, T.D. 2014 Spontaneous stochasticity and anomalous dissipation for burgers equation. preprint arXiv:1401.5541.

Eyink, Gregory L. 2011 Stochastic flux freezing and magnetic dynamo. Phys. Rev. E 83, 056405.

Eyink, Gregory L \& Benveniste, Damien 2013 Diffusion approximation in turbulent twoparticle dispersion. Physical Review E 88 (4), 041001.

Falkovich, G., Gawȩdzki, K. \& Vergassola, M. 2001 Particles and fields in fluid turbulence. Rev. Mod. Phys. 73, 913-975.

FALLER, A.J. 1996 A random-flight evaluation of the constants of relative dispersion in idealized turbulence. J. Fluid Mech. 316, 139-161.

Hentschel, H. G. E. \& Procaccia, Itamar 1984 Relative diffusion in turbulent media: The fractal dimension of clouds. Phys. Rev. A 29, 1461-1470.

Hughes, B.D 1995 Random walks and random environments, vol. 1, Clarendon.

Ilyin, Valery, Procaccia, Itamar \& Zagorodny, Anatoly 2010 Stochastic processes crossing from ballistic to fractional diffusion with memory: Exact results. Phys. Rev. E 81, 030105 .

Kurbanmuradov, O. \& Sabelfeld, K. 1995 Stochastic Lagrangian models of relative dispersion of a pair of fluid particles in turbulent flows. Monte Carlo Methods and Appl. 1, $101-136$.

Obuknov, A.M. 1941 On the distribution of energy in the spectrum of turbulent flow. Izv. Akad. Nauk SSSR, Ser. Geogr. Geofiz. 5, 453466.

Otт, S. \& MAnN, J. 2000 An experimental investigation of the relative diffusion of particle pairs in three-dimensional turbulent flow. J. Fluid Mech. 422, 207-223.

Ouellette, N.T., Xu, H., Bourgoin, M. \& Bodenschatz, E. 2006 An experimental study of turbulent relative dispersion models. New J. Phys. 8, 109.

Rast, M.P. \& Pinton, J.-F. 2011 Pair dispersion in turbulence: the subdominant role of scaling. Phys. Rev. Lett. 107, 214501.

Richardson, L.F. 1926 Atmospheric diffusion shown on a distance-neighbour graph. Proc. $R$. Soc. Lond. Ser. A 110, 709-737.

Salazar, J.P.L.C. \& Collins, L.R. 2009 Two-particle dispersion in isotropic turbulent flows. Annu. Rev. Fluid Mech. 41, 405-432.

Sawford, B.L. 2001 Turbulent relative dispersion. Annu. Rev. Fluid Mech. 33, 289-317.

SAwford, B.L., Yeung, P.K. \& HACKL, J.F. 2008 Reynolds number dependence of relative dispersion statistics in isotropic turbulence. Phys. Fluids 20 (6), 065111.

Scatamacchia, R., Biferale, L. \& Toschi, F. 2012 Extreme events in the dispersions of two neighboring particles under the influence of fluid turbulence. Phys. Rev. Lett. 109, 144501.

Shlesinger, MF, West, BJ \& Klafter, J. 1987 Lévy dynamics of enhanced diffusion: Application to turbulence. Phys. Rev. Lett. 58 (11), 1100-1103.

Thomson, DJ \& Wilson, JD 2013 Lagrangian Modeling of the Atmosphere, chap. History of Lagrangian stochastic models for turbulent dispersion, pp. 19-36. Wiley Online Library.

YEung, P.K. 1994 Direct numerical simulation of two-particle relative diffusion in isotropic turbulence. Phys. Fluids 6 (10), 3416-3428. 